\title{
HELICOBACTER PYLORI ERADICATION PHARMACOTHERAPY INDUCED PSYCHOTIC EPISODE
}

\author{
Arif Çipil ${ }^{1}$, Seda Kiraz ${ }^{2}$, Meliha Zengin Eroğlu ${ }^{3}$ \& Anıl Gündüz ${ }^{4}$ \\ ${ }^{1}$ Department of Psychiatry, Sivas Numune Hospital, Sivas, Turkey \\ ${ }^{2}$ Department of Psychiatry, Hitit University Erol Olçok Training and Research Hospital, Çorum, Turkey \\ ${ }^{3}$ Department of Psychiatry, Haydarpaşa Numune Training and Research Hospital, Istanbul, Turkey \\ ${ }^{4}$ Department of Psychology, Istanbul Kent University, Istanbul, Turkey
}

received: 10.4.2021;

revised: 14.5.2021;

accepted: 20.5.2021

$* * * * *$

\section{INTRODUCTION}

Clarithromycin is a semisynthetic macrolide used with other medicines to treat stomach ulcers caused by helicobacter pylori (HP). It has psychiatric side effects like confusion, insomnia, psychosis and depersonalization. Amoxicillin is a penicillin antibiotic used to treat bacterial infections. Antibiotic-associated psychiatric symptoms were first described 60 years ago following penicillin administration and described as psychotic symptoms (Cohen 1955). Psychosis related with antibiotics usually occures after the first antibiotic dose and dissolved within one week after the end of drug therapy. Men also seemed to have a previous psychiatric history, they were more vulnerability. The term antibiomania was adapted to describe antibiotic-induced manic symptoms which is the rare side effect of the antibiotic-use. Although the frequency of this adverse event is rare in the literature, there is a growing number of cases in patients without the previous bipolar or psychotic disorder history (Abouesh et al. 2002).

The aim of this case report is to present a case who acute psychosis evolving after the start of triple therapy for Helicobacter pylori and remitted to healthy mental status after discontinuation of the regimen.

\section{CASE REPORT}

A 45-years-old, married man with no previous psychiatric, trauma or substance-abuse history was admitted to emergency clinic because of abnormal behaviors, irritability, and suspiciousness. He had acute persecutive delusions associated with intuitive, interpretive and imaginative mechanisms. Psychomotor excitement, aggressiveness and irritability, verbal disinhibition and a denial of problems were also present.

Place, person and time orientation available, vital parameters were normal. Physical and neurological examinations were within normal limits. Complete blood count, blood glucose, detailed metabolic panel, urinalysis, electrocardiogram were normal. Computerized tomography scan of the head was normal. Additionally, his wife defined that he and his family had no psychiatric or neurologic disorders before. Medical history revealed that he had started to use triple therapy for HP gastritis five days ago, which includes lansoprazole $30 \mathrm{mg}$ once a day, amoxicillin $1 \mathrm{~g}$ twice daily and clarithromycin $500 \mathrm{mg}$ twice daily.

The patient was hospitalized to the inpatient psychiatry clinic for further exploration of this acute psychotic attack and all medications were stopped. He was extensively examined to find any organic cause for the current psychotic attack, including brain magnetic resonance (MR) imaging and electroencephalogram (EEG), which did not to reveal any abnormalities. The possible cause of this psychotic symptom attributed to an HP triple treatment(amoxicillin, lansoprazole, clarithromycin, or a combination of these drugs) since the psychotic symptoms of the patient began to diminish within a few days after cessation of the HP treatment without any antipsychotic administration. The patient returned to normal mental status within in 3 days. Adverse Drug Reaction Probability Scale (Naranjo) was administered to the patient. Total score is 7 . Total score was probably 5 to 8 , suggesting the drug reaction.

\section{DISCUSSION}

Antibiomania cases reported by the WHO and the FDA were most frequently associated with clarithromycin, followed by ciprofloxacin and ofloxacin (Abouesh et al. 2002). Since our patient received a combination of therapy (clarithromycin, amoxicillin, and lansoprazole) for HP, we have to consider the possibility that any of three (or combinations thereof) were potentially involved in the onset of psychotic symptoms. These regimens are also known to regulate approximately 8590\% cure rates of triple treatments (Hsu et al. 2014). Triple regimens may be as follows; amoxicillin, clarithromycin and omeprazole for 10 days; bismuth subsalicylate, tetracycline and metronidazole for 14 days; and amoxicillin, clarithromycin and lansoprazole approved for 10 or 14 days. Triple treatment has a wide side effect profile and the most common side effects in the gastrointestinal tract (nausea, vomiting, diarrhea, abdominal pain occur) (Hsu et al. 2014). Clarithromycin causes gastrointestinal symptoms similar to amoxicillin, but may also cause a headache and irritation. Unlike with Proton 
pump inhibitors (PPI), there have been many case reports for psychiatric consequences due to amoxicillin and clarithromycin. The Adverse Drug Reaction (ADR) Probability Scale was developed to help standardize the causality assessment for all adverse drug reactions (Naranjo et al. 1981). It is very simple and often used.

Although there have been rare reports of amoxicillin, clarithromycin more frequently associated with the antibiomania (Abouesh et al. 2002). Few cases of acute psychosis caused by amoxacillin have been reported. One patient developed mania and psychosis 10 days after the onset of amoxicillin for urinary tract infection. Symptoms ended 12 days after discontinuation of the antibiotic (Beal et al. 1986). Similarly, agitation and bizarre behavior with visual hallucinations and delusions developed in a patient prescribed amoxicillin-clavulanate for suspected pneumonia (Bell et al. 2008). All of these cases showed that acute psychiatric symptoms improved completely after amoxicillin was discontinued.

These cases prescribe drug interactions as triggers of psychosis when the patient receives multiple drugs. Clarithromycin is metabolised by oxidation and demethylation via cytochrome P450 isoenzyme (Peter \& Clissold 1992). Hypotheses have been proposed are increased prostaglandin and cortisol levels or reduced GABA concentration in the central nervous system (Abouesh et al. 2002). Inhibition of the cytochrome P450 system in multiple treatment regimens of the macrolide group may lead to this picture. Neuropsychiatric symptoms that develop during the use of clarithromycin as part of antiretroviral therapy for Human Immunodeficiency Virus (HIV) have been tried to be explained by this mechanism (Prime \& French 2001). Toxic antibiotic levels, anti-inflammatory properties of antibiotics, possibly inhibit prostaglandin E-2, GABA antagonism and N-methyl-D-aspartate (NMDA) receptor hypofunction, are possible mechanisms for this rare reaction. The latter two of which have been discussed for schizophrenia (Cohen et al. 2015). However, urinary tract infections can cause psychotic symptoms and delirium (Chae \& Miller 2015). Nevertheless, it is not known why some individuals, perhaps genetically, are more vulnerable to developing a chronic disease like schizophrenia, regardless of treatment with antibiotics. There may be a link between the immune system abnormalities seen in schizophrenia and the mechanism that causes a psychosis in individuals receiving antibiotic treatment.

It is difficult to talk about incidence-prevalence rates of antibiomania because of limited data. There are still uncertain points about the pathogenesis of these conditions. This case report may be useful for both mental health and other health professionals to be more cautious about the antibiotic-associated psychiatric problems including psychotic episodes. Physicians and other health professionals should be aware of these psychiatric side effects of antibiotics.

\section{Acknowledgments: None.}

\section{Conflict of interest: None to declare.}

\section{Contribution of individual authors:}

Arif Çipil: study conception and design, acquisition of case history.

Seda Kiraz: interpretation of data, drafting and revision of the manuscript.

Meliha Zengin Eroğlu: first draft.

Anıl Gündüz: study design, drafting of the manuscript.

\section{References}

1. Abouesh A, Stone C, Hobbs WR: Antimicrobial-induced mania (antibiomania): a review of spontaneous reports. $J$ Clin Psychopharmacol 2002; 22:71-81

2. Beal DM, Hudson B, Zaiac M: Amoxycillin-induced psychosis? Am J Psychiatry 1986; 143:255-256

3. Bell CL, Watson B, Waring WS: Acute psychosis caused by co-amoxiclav. BMJ 2008; 337:a2117

4. Chae JH, Miller BJ: Beyond urinary tract infections (UTIS) and delirium: a system-atic review of UTIs and neuropsychiatric disorders. J Psychiatr Pract 2015; 21:402-411

5. Cohen SM, Tsien RW, Goff DC, Halassa MM: The impact of NMDA receptor hypofunction on GABAergic neurons in the pathophysiology of schizophrenia. Schizophr Res 2015; 167:98-107

6. Cohen SB: Psychosis resulting from penicillin hypersensitivity; report of a case and review of the literature. Am J Psychiatry 1955; 111:699-702

7. Hsu PI, Wu DC, Chen WC, Tseng HH, Yu HC, Wang HM et al. Randomized controlled trial comparing 7-day triple, 10-day sequential, and 7-day concomitant therapies for Helicobacter pylori infection. Antimicrob Agents Chemother 2014; 58:5936-42

8. Naranjo CA, Busto U, Sellers EM, Sandor P, Ruiz I, Roberts EA et al.: A method for estimating the probability of adverse drug reactions. Clin Pharmacol Ther 1981; 30:239-245

9. Peter DH, Clissold SP: Clarithromycin. A review of its antimicrobial activity, pharmacokinetic properties, and therapeutic potential. Drugs 1992; 44:117-164

10. Prime K, French P: Neuropsychiatric reaction induced by clarithromycin in a patient on highly active antiretroviral therapy (HAART) Sex Transm Infect 2001; 77:297-298

\section{Correspondence:}

Seda Kiraz, $M D$

Psychiatry Department, Hitit University Erol Olçok Training and Research Hospital

Çorum, Turkey

E-mail:drsedakiraz@gmail.com 